Servicio de Tisiología

"Hospital Arriarán"

Sanatorio "Los Guindas"

de' Consejo de Defensa del Niño.

\title{
LA ATELECTASIA PULMONAR EN EL NINO Y SUS RELACIONES CON LA TUBERCULOSIS PRIMARIA
}

\author{
Por los Dties. JORGE PEÑA CERECEDA, ERNESTINA PEÑA D. \\ Y LUCILA CAPDEVILLE
}

(Contintación)

OBSERVACION CLNICA N'. 19. Servi:io de Tuberculnsis. 3459.... H. Arriarán.

J. A.-8 años. Ingreso: 3-XI-39.

ANTECEDENTES.-U'n tubercaloso que frecueniaba la casa murio a consccuencias da su tesion pulmonat bace un año. Coupeluche an 1934.

ENFFERMDAD ACTUAL.-En febrero di 1939 ingresa al Servicio de Medicina por un cuadto sospethado de dertame pleural izquierdo.

Mantoux al l por míl: positivo. Sedimentación: 20-30 mm/h. Pes: $20.500 \mathrm{~kg}$.

Al examen fisico, se encuentra un niño de regular estado general, pálito y at examen pulmonar, submacidez y cisnitición de: murmullo vesiculat en el tercio inferiar izquierdo. La radioscopía informa que existe anja sombra densa homogénea. que ocupa todo el hemitórax jzçuicrdo. el que está inmovil. Camipo derebo normal. Mediastino en posición normal.

Nuevo roves más tarde el probable detrame se ha rabsotbido, y la ratios. copix indica sembra de mediara intensidad, que ocupa la mitad superior del pulmón izquierdo. Sombra trene matginat: izquierda.

Al mes siguiente la sontbra pulmonar sn ha raducito más aún. Contirín controlindese. a pesar que presenta mug buen estado general y está afebrit. Scdimen1stion: $15-20 \mathrm{~mm} / \mathrm{h}$. Peso: $20 \mathrm{~kg}$.

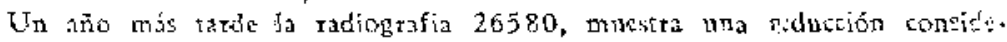
table de $l:$ inagen. persistiendo algunas sombras eth formas de rractus de indw. ración en h región jnterclejdo hiliar izquietda y reinozamicnto in bestructura dal hilio. Campo derecho. normal.

Comentaric.-Ingresa por un probable derrame pleural izquierdo que se reabsorbe rápidamente, quedando a ia radio- 
grafía sólo una imagen de infiltración en la mitad izquierda. Un año después, la imagen se borra, dejando un campo de induración en el tercio superior de ese mismo lado. La evolución indica que se trata de una atelectasia masiva izquierda $y$ no de un derrame como se pensó en un çomienzo. Este diagnóstico se basa en la falta de signos claros de derrame y en la reducción rápida de la imagen radiológica, en un principio parcial y posteriormente de toda el área pulmonar.

OBSERVACION CLINICA N." 20,-Servicis Tuberculosis. 438\%,-H. Artiarín.

A. P.-3 años.-lngreso: $15-X-39$.

ANTECEDENTES.-El abuelo paterno falleció de tuberculosis en el año 1938 y visitaba con frecuencia la casa. Coqueluche en 1937.

Marleux al 1 por mil: positivo. Sedimentación: $25-30 \mathrm{~mm} / \mathrm{h}$. Pess: $8 \mathrm{~kg}$.

At examen encontramos una niña de regular estado genetal. Afebril, serflaquecida, sin tos ni expectoración.

Al examen pulmonat no hay signos anormales.

La radiogtafía 14597 , del $6 \times 39$, da una sombra muy densa $y$ homo. génez. que otupa gtan parte del campo pulmona: izquietdo. quedando libre la región costo-diafragmática.

Continúr en control, con butn estado general. Afebril.

Dos mises mas tarde una radiografia indica que la ombra se ba redacida visiblemente, sólo compromete ei tercio superict. Este cuadro pulnonar no su. fre vatiaciones por espacio de un año, durante el cual se manting en muy buten e:tado general. Sedimentación: $5-8 \mathrm{~mm} / \mathrm{h}$. Peso: $10 \mathrm{~kg}$. Examen pulmonit: Hegativo.

La ratiografía 31556, del 8-1X-41. o sea 2 años despuis de la iniciacicin

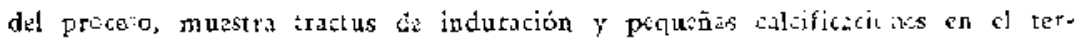
cio superior izquitrdo. Calciiticaciones de los gavglios faraturquedes. Resto: normal.

Sigue binn. Las contro'es clinitos y tadiológitos posteric es siempre han us sultado Hegativos.

Comentario.- Se trata de una atelectasia de la mayor farte del hemitórax izquierdo a juzgar por la ausencia de sintomas clínices y por su desaparición rápida (5 meses).

Analizaremos aqui también los casos en que las sombras comienzan a borrarse de la periferia hacia el centro, quedindo limitadas a las regiones vecinas a1 mediastino. Tal sucede por ejemplo en las atelectasias del lóbulo superior, que se borran en forma de abanico, persistiendo una delgada sombra paralela a la cadena de los ganglios paratraqueales. Tenemos 6. mo ejemplo la observación sigurente: 
OBSERVĂCION CLINICA N.? 21.-Servicio de Tubarculosis, 460-D --I1. Artiarán.

M. S.-5 años,-Ingre:0: $1 .^{n}-$ II-39.

ANTECEDENTES,--Madrc falleció de tuberculosis puimonar, en dicirmthe da 1938. Sarampión hace 4 años.

ENFERMEDAD ACTUAL-Consulta por mal cstado gencral, desimiento, inapat?ncie. Al cxametn ancontramos un niño pálido, sin tos ni expertotación. Afebril.

El examin puimonar da una respiración tuda en el cercio superior $d:$ recho.

Mantoux al 1 por mil: positivo. Peso: $15 \mathrm{~kg}$. Sedimenclación: $41-79$ $\mathrm{mm} / \mathrm{h}$. Baciloscopia: negativa. Cultivo: negativo.

La radiografia 12565, del 1 , 9 -39 (figura 14-a), muestra una sombra grandi y homogina que octpa el verrice 'y la region infraclavicular derecha. Resto liber

Durante sa enfermedad praienta ua vaticela intercurrente, que no influte majormente en sp evolución.

La radiografia 17300, del 8-IIl-40 (figura 14-5), mucstra que la sombra đel tátcio superior ha Jesaparecide. Quedan algunos trấclus indurados a nivinl de lu región paxatraqual derecha.

Sedimentacióri: $12.39 \mathrm{~mm} / \mathrm{b}$. Peso: $16.500 \mathrm{~kg}$.

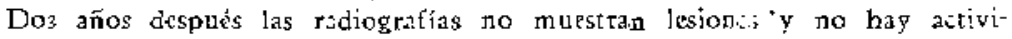
dad del procsso. Bnen estado genetal, Afobri!.

Examen pulmonar: negativo. Sedimtntation: $6-12 \mathrm{~mm} / \mathrm{h}$, Peso: $10 \mathrm{~kg}$.

Comentario.- Se trata de una atelectasia del torcio $515-$ perior derecho, que se borra diez meses después de la pirifcria al centro. Esta lesión ha sido provocada, seguramente, por ganglios pequeños de la región paratraqueal derecha, que se alcanzan a distinguir en las újtimas radiografías.

En lo que se refiere a la situación y compromiso de las cisuras, podemos decir que en nuestros casos comprobamos que las cisuras, especialmente la horizontal, se aptecian desviadas en sentido oblicuo, en mayor o menor grado, según 1. . Extensión de la atelectasia.

Con la regresión del proceso recuperan su posición primitiva en los casos de evolución rápida, pero cuando se pro duce 12 induración, estas modificaciones se hacen definitivas. Así tenemos la obsetvación siguiente.

OBSERVACION CLINICA N," 22.-Sanatorio "Los Guindos". 616-B. N. L.—- años,-Ingreso: 10-IV-41.

ANTECEDENTES.-No se precisa el contagio. Sârampión y coquẹucba c) 1940. 
ENFERMEDAD ACTUAL.-Ingtesa per decaimiento, palidez y anor xi.u. Al examen encontramas una niña de aspecto más o menos regilar, con isthas tos, sin de:garro. Afebril.

El exarien pulmonar da estertores gruesos en la base derecha.

Sedimancacion: $74-100 \mathrm{~mm} / \mathrm{h}$. Pcso: $17.500 \mathrm{~kg}$. Baciloscopia: negaliva.

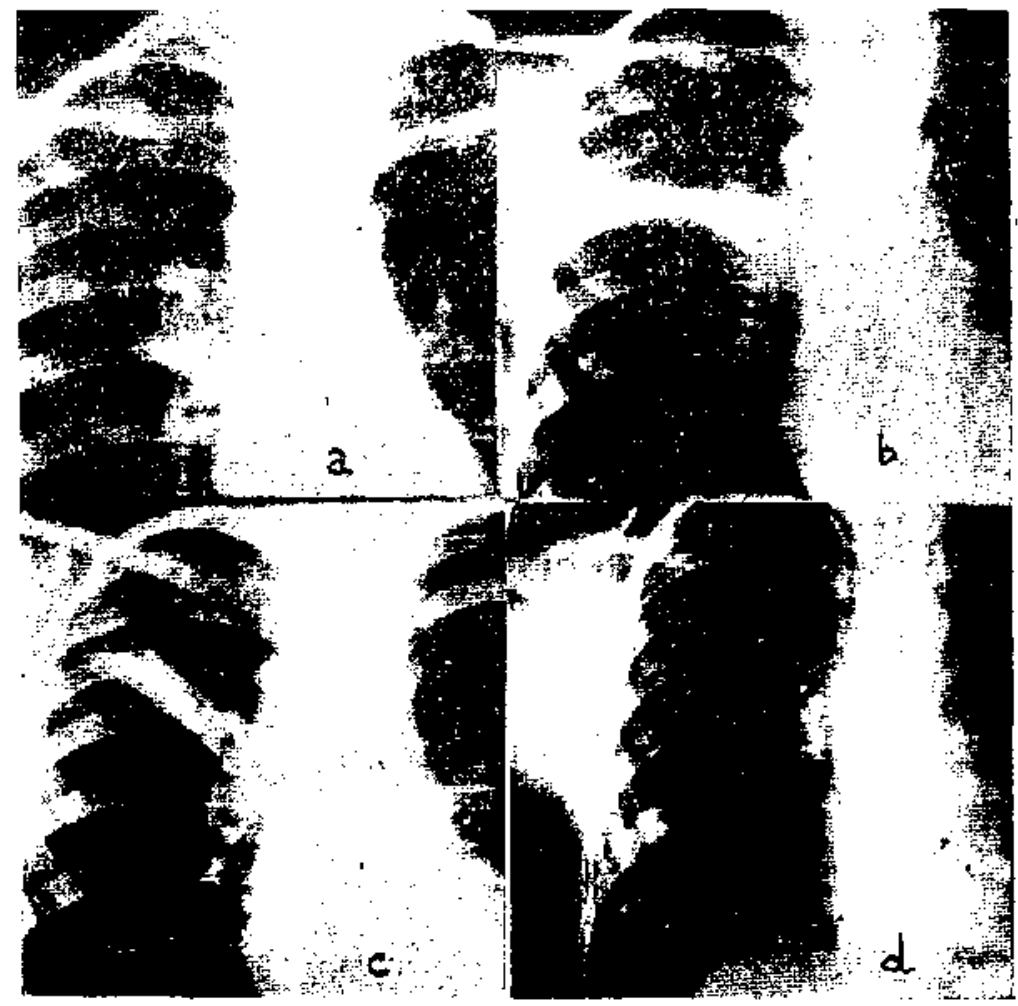

FIGURA N. 20. - OBSERVACION N." 22

a) Adenopatia biliar derecha. Reforzamiento de la trama en la región intercleido hiliar de ese 1ado; b) Sombra en forma de faja en el tercio medio detecho: e) Ia sombra ha dasalparecido. So aprocia nra elevación de la cisura hacia arriba y hacia afueca; d) El medio de contraste permite apreciar dilataciones bronquiales residuales en el sitio de las antiguas lesiones.

La radiografía 9666, del 7-III-41 (figara 20-a), da una sombra Jensa co el hilio derecho con caracteres de adezopatía. Reforzaminto de la trama pulmonar en la región esciapnlar e intercleidobiliar derecha. Lado izauierde. normal,

La radiografia 9733 del $3-[V-41$ (́́igura 20-b), da sombra en forma de faja horizontal en la unión del tertio superior con el retcio medio derecho. 
Continứa en control. Ocho mesea después, el examen pulmonar da alguncs roncus diseminatos en ambos campos. Existe buen estado general. Afebril.

Sedimentación: $25-50 \mathrm{~mm} / \mathrm{h}$.

La radiogtafía 11262 (figura 20-c), indica que las sombras del hemito. rax derecho han desaparecido, persistiendo una sombra alargada con aspetio de induración residual en la región peribiliat superior derecha. Rexto: norma!.

$S=$ continúa observando por espacio de 2 años, sin apreciarse a rabios d: iniportancia.

En marzo de 1943, practica una BRONCOCRASIA con lipicdol, y ta radiografia 28654 (figura 20-d), indica la ptzis:ncia de dilataciones y deformaciones bronquiales residuales, en correspondencia con los torritorios antiguamenIi ${ }^{1}$ exionados.

Ccmentario.-En esta observación podemos aprecia: en frimer lugar, la aparición de una atzlectasia en forma de fain en la parte inferior dén. lóbulo superior, y en segundo lusar. ex formación de dilataciones bronquiales en la región hiliar y a lo largo de la cisura horizontal, que há guedado fuertemen. te desviada hacia arriba.

Por último, debemos considerar los procesos residualcs. que como lo denuestra la observación anterior, tienen much: interés por las sacuelas y los trastotnos a que dan origen.

Gran numaro de atelectasias legan a reabscrberse $\mathrm{com}$. fletamente (Observación 17) y ouras dejan zonas de tojide fibrono (carnificación de Laennes): pero junto a estas fibro. sis quedan a menudo dilataciones bronciaiales determinads tor ateracicnes dz las paredes, que se revelan al examen mdicgrafico, como imágenes en panal de abejas o en forme di Jiners divergentes e irregulares que corresponden a las por. dia tronquiales engtosadas. Estas alieraciones se ven con mayor frecuencia en el ángulo costodiafragmático deracho 110 . bulc mediol. en las regiones mediastino-diafragmáticas y on

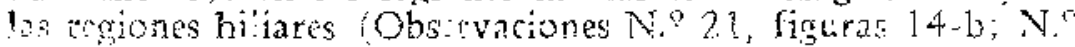
24. figura $21-b$, y N." 28, figura 23-b).

\section{Signe: bronescópicos}

Generalidades.-Srgún la definición de Clapvaliet Jackson y Chevalier L. Jackson (17), se entiende por endos:onia. el examen de una caridad o trayecto del auetro humano m:diante un endosccpio, que, según los casos, podrá ser una !arinccecopia directa, una esofagescopía, gastroscopía, bronrosiopía, etc. 
La broncoscopía se emplea en el diagnóstico y tratamiento de algunas enfermedades, como las estenosis, las obstrucciones o tumores que compriman las paredes bronquiales. Además se la emplea para la extracción de secreciones de los bronquics o para tra obtención de biopsias, etc.

En general la broncoscopia no requiere anestesia gene ral: basta la anestesia local de la faringe con novocaína al $1 \%$ o con cocaína al 10\%. Este último método ha sido empleado en todos nuestros casos.

Indiciciones.-Puede decirse quie las indicaciones más comunes en el niño son: 1) Atelectasias; 2) Bronquiectasias: 3) Abscesos agudos: 4) Obstrucciones bronquiales, ya sea por cuerpos extraños o por secteciones; 5) Para la introducción de sustancias de contraste en el árbol respiratorio. como tiempo previo de la broncografía; 6) En ciertos casos de laringotráqueobronquitis agudas, $y$ en genetal!', en todas las enfermedades que produzcan disnea o estridor, sin que se encuent to una causa precisa que las determine.

En general, la broncoscopía puede practicarse en casi ta. dos los cacos, aun los más graves, excepto en niños menomet de 1to año, en los cuales la exploración broncosópica deb: pracicatse con sumo cuidado y durante un tiempo muy corto. rara no producir edema de ía glotis.

La broncencopia como métcdo diagnóstico en las atel: tasias di nirio. tanto agudas como crónicas as, a nuestro juicio. uro de los más impcrtantes junto con el eximen racio lógico.

En ofecto. el broncosccpio nos permite explo ar las vise reptacias desde la tráquea hasta los brenquios de primeir y segundo orden.

Según Gaecia Arrazuria (25), los tipos mis frecusites: de lisicnes traqueobrónquicas tuberculesas soli las siguicntes:

a) Ulceraciones de la mucosa, superficiales o protunGas:

b) Proliferación granulomatesa de ia mucosa:

c) Hipertrofia de la submucosa, sin altevición de la murces:

d) Fibrosis o estadio fínal de toda lesión bronquia!.

Este autor considera que la congestión de la muscsa bronquial es la fase previa para cla instalación de un proce. sc ulceroso o infiltrativo.

Esta clasificación corresponde más bien a los adultos. Por nuestra parte, hemos observado que en los niños predonina de preferencia, lia congestión y el edema de la mucosa. 
En pocos casos se observan ulceraciones que pueden al. canzar hasta la perforación de la pared (Obs. 7, figura 13). Más raramente existen proliferaciones granulomatosas.

La fibrosis, con estrechamiento del lumen bronquial, se otserva regularmente en las fases avanzadas de la inflamación de las paredes bronquiales y se produce principalmente por la induración de ganglios tuberculosos circunvecinos.

Un hecho de gran importancia es la presencia de secreciones mucosas, mucopurulentas o purulentas en todos los ca. sos de atelectasias agudas y que alcanzan a cantidades de 150 a $200 \mathrm{cc}$. Estas secreciones tienen un aspecto viscoso, adheren. te y ccupan totalmente un bronquio de mayor o menor calibre, las cuales es preciso extraer para hacer la observación de la mucosa lesionada. Este solo hecho basta, en muchos casos, para hacer el diagnóstico de atelectasia por obstrucción en los casos agudos. En las formas crónicas o subcrónicas las secreciones bronquiales disminuyen considerablemente, hasta desaparecer casi por completo en las atelectasias antiguas.

La forma que adquieren thas obstrucciones bronquiales por estos exudados. varía según los cascs: en algunos, (y esto sucede especialmente en los períodos agudos), reviste la forma de una hendidura vertical o semilunar, en ottos aparece en forma dentada $y$ por último en forma de anillo circulat aue estrecha el lumen del bronquio.

El estudio seriado del árbol bronquial por medio de la broncoscopía nos permite obsetvar esta desaparición de los exudados, al' mismo tiempo que la regresión de la tumefacción correspondiente de la mucosa. En algunas ocasiones se puede apreciar una reagudización de la inflamación de la pared bronquial, junto con el aumento considerable de las secteciones, lo que se traduce, al examen radiolb́gico, por un aumento de la sombra atelectásica. Estas últimas formas son frecuentes en el curso de enfermedad's infecciosas, especialmente la coqueluche (como en la observación N. 15) y en íos casos de niños muy pequeños, raquíticos o con diáresis exudativa.

Exponemos a continuación nuestras observaciones sobre broncoscopía, que en total ascienden a 16.

Cuatro de ellas llegaron por diversas circunstancias a la mesa de autopsia (Observaciones N.os $1,2,3$ y 4) y han servido para comprobar, de una manera indiscutible, la importancia de los datos que se obtienen por medio del método broncoscopico. 
OBSERVACION CLINICA N. ${ }^{g}$ 23.- Servicio de Infecciosos. 5045 -J. A.-1 año 2 meses.-Ingreso: $5 \cdot \mathrm{XI}-42$.

H. Arriarín.

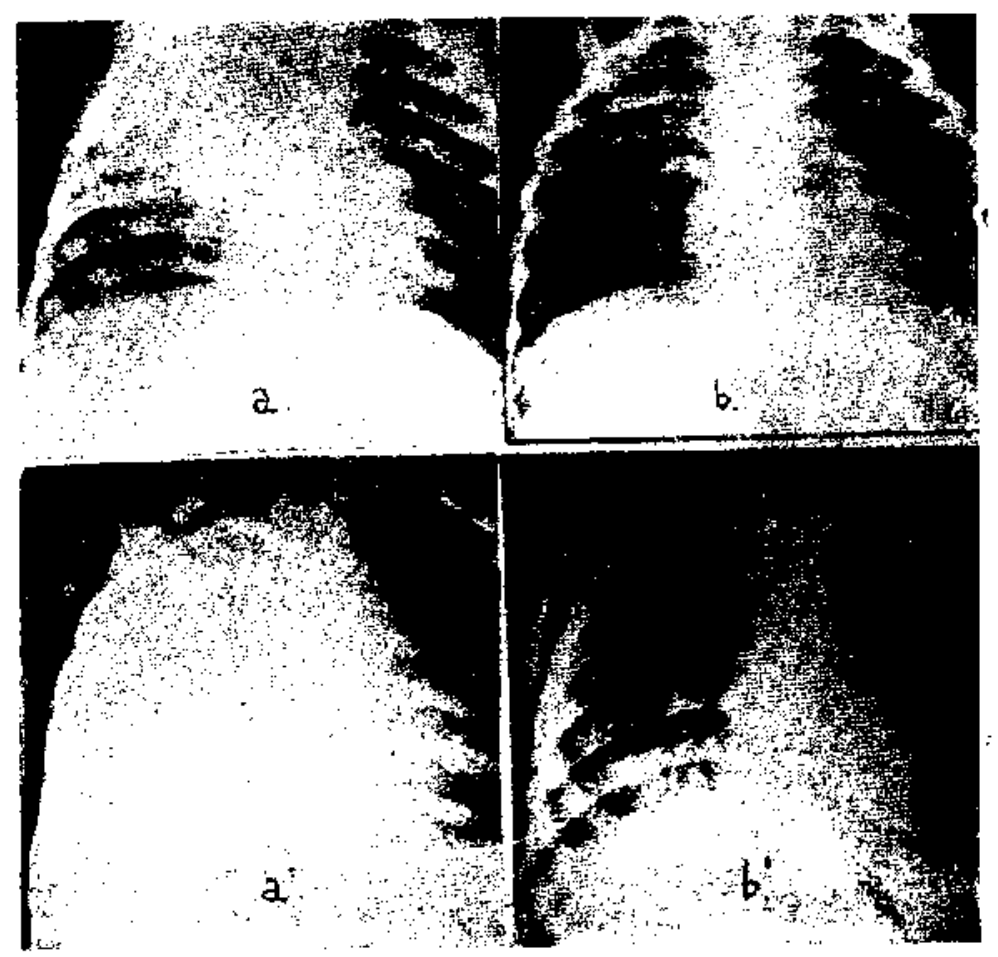

FIGURA N: 21. - OBSERVAICION N. 23

a) Sombra de atelectasia de los dos cercios superiores derechos; b) ĺa sombra ha desaparecido. Quedan restos de induraçión que simatan una falsa caverua. IFTGURA N.21, C OBSERVACION N: 24

a) Inuaren de atelectasia masiva derecha con desviación dz: mediastino haxita ese mismo lado. Pequeña zona chata marginal inferior derecba; b') La sombsa ha desaparecido easi en su totalidad.

ANTECEDENTES-Ingresa al Servicio de Infecciosos, con el diagnóstico de difteria. Presenta fiebre alta, tos espasmódica, ronquera y poco desplués disnea. Se colocan 15,000 unidades de suero antidiftérico. El examen laríngco indica que las cuerdas vocales están rosadas y cubiertas de pequeñas fotmacion:s superficiales. del tamaño de un milimetto, zon fondo tojo y que solevantan la mucosa. No se encuentran falsas membranas. Cultivo para bacilo de Loeff!er negativo en la secreción faringea, Se encuentran en cambio, espirilos y neumococos. 
E1 tratamicnto por el suero no dió resultados, porque el niño siguió en las mismas condiciones y sa agravó más tande por la aparición de ana otitis media suparada.

Mantoux al 1 por mil: negativo. Bacilostopia: negaliya.

Al examén pu'monar se encuentra submacidez de la mitad supetior dere cha, con silencio respiratorio. Estertores gruesos, escasos, diseminados.

Sedimentación: $76-100 \mathrm{~mm} / \mathrm{h}$. Peso: $6.900 \mathrm{~kg}$.

A la radiografia 36683, (figura 21-a), se encontró una sombra densa $y$ homogénea, de límite inferior difuso, con caracteres de infiltación pulmonat. que ocupa los dos tercios superiores del lado derecho. Velo tenue en al tercir medio e infatior del mistnc lado. Campo izquierdo: norma!.

BROYCOSCOPIa-(3-X-42): Se observa, en cl espolón traqusin y az la purte posteriot del bronquio derecho, une tumefacción ds la racosa (D:. Pa. rada).

Diez dias más tarde, la radiografia 37285, (figura 21-b), muestra $1_{3}$ riahorción, ien gran parte. de la scmbra del iado derabo, persi-tiendo son. bras tenaéz e irtegu'ares en el tercio superior y una imagen clara, anulat, sospechesa da excayacióa, en la región escapular superior.

El estado gencral del niño va poco a poco mejorando, está afebril, tistit tuen apetito, no tose. La sedimentación se ba, normalizado y los últimaí cun: trols radiográficos no muestran lesiones.

Comentario,-Este caso tiene interés desde ell punto de vista del diagnóstico diferencial con la difteria (como lo veremos más adelante en la Observación $\mathrm{N}^{\circ} 34$ ), ya que es frecuente el comienzo brusco, con síntomas de asfixia, en un buen númerc de obstrucciones bronquiales.

La lesión que presentó a nivel de las currdas vocales. tuvo, con toda probabilidad, un origen espiril ar ya que colić al tratamiento con bismuto.

OBSERVACION CLINICA N." 24.-Servirio de Tubertulosis. 56634. - H. Arriarán.

L. M.-2 años 6 meses.--Ingreso: $25-\mathrm{IX}-42$.

ANTECEDENTES, - Visitaba a un vectro que padecta de tuberculosis pümozar. Varicela hace un año. Eitados catarrales agudes de repetición. Sa. rampión en agosto de 1942.

ENIFERMEDAD ACTUAL.-Después de esta última enfermedad, quist.t decaida, inapetente, con transpiración nocturna $y$ tos intensa.

Al examen se encaentra una niña enflaquecida, con temperatara de $38^{\circ} \mathrm{y}$ tos intensa.

Peso: $11 \mathrm{~kg}$. Sedimentación: $76-128 \mathrm{~mm} / \mathrm{h}$. Baciloteopía: negativa. Mantoux al 1 por mil: positivo.

Al examen pulmonar micontramos: submacidez y disminución del orurmullo vesicular en el campo drecbo. 
Radiggrafía: 22-IX-42 (36.584, fiqura 21-a), matsera combra irrepatis gue acupa casi todo el hemitórax derecho. Sombra marginal densa, en forma a faja, con aspecto de derrame pleural cn la región marginal izquierda.

Se llega al diagoóstico de EMPIEMA pleural iscquictido, por lo qui st: leva al Servicio de Ciragía para nna intervención.

Como la panción resultaca blança, se desiste de la opetación y se contincia en observasión. La ficbre dessparece una semana después: peto los sinternas pulmonares se mantienen igualis.

S:dimentación: $60.105 \mathrm{~mm} / \mathrm{h}$. Paso: $10 \mathrm{~kg}$.

Radiografía del 5-X.42 (36765, Iigura 21-b). Ha desaparecido casi m zompleto la sombra derecha. Sólo queda una imagen alargacta en la unióa dei tercio medio y superior dereches. Pequena adenopatia pararraciatal derecha. Fa. quipkuxitis maxginal izquierda.

Contrac no coqueluche en el Servicio, que evoluciona durante 2 mesc.,

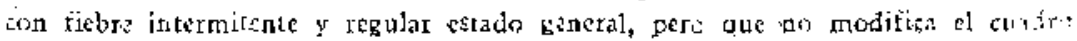
pu'monat.

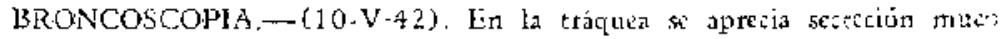

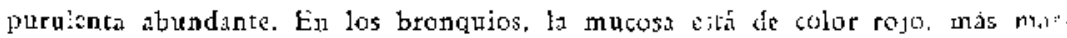
$\therefore$ do que en la tráqua $y$ con la misma secreción mucopuruidata. En il pres quio dercho. aśemás. a 2 ó 3 centimeltos de la bifurcación. se encuentia wa, imagen an forma de mamelones de la mucosa, cn númaro d: 3 a 4 , colocados en distinta; p'inos.

I.a interpretación qu: pucde dares a esioe mam:lones, es que stan un proceso de granulación en una ulceración de la mucosa (Dr, Paradal

La niña ha seguido en buenas condiciones generales. a.febril $y$ con ts casa tos. Las costroles radiológico- posteriorss, no indiean ninguna vanaron ie.pectc a las ya anotadis.

BROFCOSCOPIA.-(7-YI-43). Igual a la anterior, Se extirpan jigunts Fiqueñas granulaciones (Dr. Parada).

L: control radiológico despues de la broncoscopia no nuestra variacione:.

Comentario. - Se trata de un lactante, con antecedentes de afecciones catarrales agudas de repetición que, a raíz de uit sarampión, tiene un cuadro agudo febril, con una opacidad casi total de! hemitórax derecho, que hace pensar en un empiema: sin embargo, el estudio radiográfico y la evolución clínica, crientan el diagnóstico hacia la atelectasia.

El curso posterior de la enfermedad viene a comprobar este diagnóstico. En efecto. la sombra atelectásica desapartece casi por completo en pocos días, quedando visible la imagen de una pequeña adenopatía paratraquea! dereina.

La broncoscopía practicada 7 meses después de su ingreso confirma el diagnóstico, ya que revela la presencia de mamelones de la mucosa del bronquio derecho. 